\title{
Expression of keratinocyte growth factor (KGF) and its receptor in a middle-ear
}

cavity problem.

Tomomi Yamamoto-Fukuda, MD; Haruo Takahashi, MD; Takehiko Koji, PhD

Department of Otolaryngology-Head and Neck Surgery (TY-F, HT), Department of Histology and Cell Biology, (TK), Nagasaki University Graduate School of Biomedical Sciences, Nagasaki, Japan

Running head: KGF expression in a cavity problem

Address for correspondence and reprints requests:

Tomomi Yamamoto-Fukuda, MD

Department of Otolaryngology-Head and Neck Surgery,

Nagasaki University Graduate School of Biomedical Sciences,

1-7-1 Sakamoto, Nagasaki 852-8501, Japan

Phone: $+81-95-819-7350$

Fax: $+81-95-819-7352$

E-mail: tomomiYF@nagasaki-u.ac.jp

Financial disclosure: This study was supported in part by a grant from the Naito Foundation (to T. Yamamoto-fukuda)

Conflict of Interest: The Naito Foundation (Tokyo, Japan) 


\begin{abstract}
Objective: To investigate the pathogenesis of one of the most troublesome conditions following ear surgery, a middle-ear cavity problem.
\end{abstract}

Methods: Keratinocyte growth factor (KGF) and its receptor (KGFR), the ratio of proliferating epithelial cells using Ki-67, and the extent of infiltration of B cells and T cells were examined immunohistochemically in 10 ears with a cavity problem, 70 ears with cholesteatoma and 8 ears with normal skin at the retroauricular incision.

Results: KGF was positive in $40 \%$ of cavity problem specimens, $37.5 \%$ of normal skin specimens, and was positive in $88 \%$ of cholesteatoma specimens (cavity problem versus cholesteatoma, $\mathrm{p}=0.0004)$. The positive rate of KGFR in the cavity problem group $(33.8 \%)$ was between those in cholesteatoma $(60 \%)$ and normal skin $(0 \%)$. In contrast to the cholesteatoma specimens, a significantly smaller number of Ki-67 labeling index (LI) was detected in the cavity problem specimens. B cell LI was significantly higher but $\mathrm{T}$ cell LI was significantly lower in the cavity problem specimens than in the cholesteatoma group.

Conclusions: Our present study indicated that the discordance of paracrine action between stromal KGF and epithelial KGFR with a large number of infiltrating B cells may play an important role in the pathogenesis of a cavity problem. 
Key words: cavity problem, keratinocyte growth factor (KGF), KGF receptor (KGFR), Ki-67, B cell, immunohistochemistry

Level of Evidence: N/A. 


\section{Introduction}

The ultimate goal of cholesteatoma surgery is the complete eradication of the disease for the health and safety of the middle ear. Over the years, this has been achieved consistently though a canal-wall-down, open-cavity tympanomastoidectomy [1]. However, despite competent surgical techniques and careful revision of the cavity, many patients continue to have postsurgical ear problems, such as discharge [2]. The so-called "cavity problem" is likely exhibited by a small meatus, behind which can be found a high facial ridge, partially removed posterior and superior canal walls, a partially removed lateral attic wall, a deep mastoid tip cavity, no tympanic membrane or a tympanic membrane perforation, chronically diseased mucosa, and active mucopurulent dranage [3]. Mastoid obliteration using various materials has been described [4 - 7] to achieve drying of the mastoid, but troublesome granulation often developed even over the newly-formed canal wall [8]. Presently, a cavity problem that continues to involve persistent otorrhea and granulation may possibly be an after-effect of mastoid surgery.

Keratinocyte growth factor (KGF) is a mesenchymal-cell-derived paracrine growth factor that specifically stimulates epithelial cell growth. It is supposed to be secreted from fibroblasts mainly in the stroma and bind to KGF receptor (KGFR), which has been detected only on the surface of epithelial cells $[9,10]$. Werner et al. [11] showed KGF was expressed in normal skin and was induced upon injury. Furthermore, they indicated that KGF was predominantly expressed in stromal cells below and at the edge of the wound, and that KGFR was detected in the epidermis [11]. They concluded that a KGF-mediated paracrine interaction may be important for 
the migration and proliferation of epidermal keratinocytes seen during wound healing [11]. Staiano-Coico et al. [12] also indicated that KGF had marked effects on the epithelialization and healing of wounds.

In the previous study, focused on KGF, we demonstrated that co-expression of KGF and KGFR seemed to induce the proliferation of epithelial cells in the primary cholsteatomatous chronic otitis media (COM) as well as in its recurrence $[13,14] . \quad$ In conclusion, we suggested the possibility that co-expression of KGF and KGFR plays an important role in the transitional stage from non-cholesteatomatous COM to cholesteatoma [15].

We believe that, in order to know the pathogenesis of the cavity problem, e.g. whether it is a proliferative or defective lesion in wound healing for its prevention and treatment, comparative analysis of KGF and KGFR between the cavity problem and cholesteatoma seems important. But, to the best of our knowledge this has not been thoroughly studied yet. One of the aims of this study was to compare the activity of KGF and KGFR and investigate the difference between them in proliferative activity by Ki-67.

In previous studies, both the cholesteatoma and COM specimens appeared to contain a predominant population of chronic inflammatory cells, including lymphocytes, monocytes, and plasma cells $[16,17,18]$. No clear pathologic difference was demonstrated between these entities. However, we indicated that $\mathrm{T}$ lymphocytes, usually predominant during the acute inflammatory process, were thought to produce several cytokines that enhance the proliferation of epithelial cells in cholesteatoma [13, 
15]. On the other hand, B cells produce antibodies in damaged tissues, and are engaged in the process of wound healing [19] and some chronic inflammatory diseases $[20,21,22]$. In this study, we also investigated the difference in the population of B and T lymphocytes between a cavity problem and cholesteatoma. 


\section{Material and Methods}

\section{Patients and Specimen Harvest}

We performed a retrospective study. The subjects in the study were 80 patients (80 ears) who underwent ear surgery at the Department of Otolaryngology-Head and Neck Surgery, Nagasaki University Hospital between February 1998 and March 2010 (Table 1). We first chose patients who had a history of mastoid surgery for chronic ear disease with or without cholesteatoma, and then carefully confirmed the diagnosis with a pathologist. We then selected cases who presented with a cavity problem or cholesteatoma. Study subjects consisted of 10 patients (4 males and 6 females) with a cavity problem (10 ears) and 70 patients (40 males and 30 females) with cholesteatoma (70 ears). In 8 ears ( 4 male and 4 female) of the 70 study subjects with cholesteatoma, a small piece of normal skin was harvested during surgery. Seven of the 10 cavity problem cases had undergone previous surgery because of cholesteatoma, and 3 cases had undergone a previous surgery due to non-cholesteatomatous chronic otitis media. All of the cavity problem cases were experiencing discharge and granulation at the time of surgery. According to the pathologist, all of the cases in the cholesteatoma group were diagnosed with otitis media and cholesteatoma. Four cavity problem cases were diagnosed with chronic inflammation; one cavity problem case was diagnosed with severe inflammation and 5 cavity problem cases were diagnosed with granulation tissue. The age of the patients ranged from 27 to 71 years (average: 52 years) and from 2 to 85 years (average: 42 years) in the cavity problem and cholesteatoma groups, respectively. In the cholesteatoma group, specimens were harvested from the site that contained both epithelium and connective 
tissues that were in a matrix with connective tissues in the cholesteatoma. In the cavity problem patients, granulation tissue was harvested with the surrounding epithelium, to the extent possible. We harvested epithelial lesions with the connective tissues, and troublesome granulation tissues even developed over the newly-formed canal wall in the cavity problem specimens as possible. As a control, a small piece of normal skin was used. It was impossible to collect a normal skin specimen in the cavity problem group because only granulation tissue was removed with the surrounding epithelium. So, a small piece of normal skin was collected either from the cartilaginous portion of the external ear canal or from the edge of a retroauricular incision in 8 cholesteatoma patients.

The study protocol was approved by the Human Ethics Review Committee of Nagasaki University School of Medicine and signed informed consent was obtained from all the patients or their guardians for this study.

\section{Tissue Preparation}

All specimens of the cholesteatoma, cavity problem, and normal skin were used for enzyme immunohistochemistry. Each of the specimens was fixed overnight in $10 \%$ buffered formalin at room temperature (RT) and was embedded in paraffin. Serial sections were cut at $4 \mu \mathrm{m}$ thickness and were then placed onto 3-aminopropyltriethoxysilane-coated glass slides (Dako, Tokyo, Japan). Adjacent sections were stained with hematoxylin and eosin, and were processed for immunohistochemical analysis of KGF, KGFR, Ki-67, CD20cy, and CD45R0. 


\section{Biochemicals and chemicals}

3, 3'-diaminobenzidine-4HCl (DAB) was purchased from Dojin Chemical Co. (Kumamoto, Japan). Bovine serum albumin (BSA, minimum 98 \%), Brij 35 and Triton X-100 were purchased from Sigma Chemical Co. (St. Louis, MO). All other reagents used in this study were purchased from Wako Pure Chemicals (Osaka, Japan) and were of analytical grade.

\section{Immunohistochemistry for KGF and KGFR}

Enzyme immunohistochemistry was performed to examine the expression of KGF and KGFR in tissue sections. Polyclonal antibodies against KGF and KGFR were prepared by immunization of rabbits against synthetic peptides in cooperation with Nichirei Co. (Tokyo, Japan). Sections of 69 cholesteatoma specimens, 10 cavity problem specimens and 8 normal skin specimens were used for KGF staining. Sections of 62 cholesteatoma specimens, 9 cavity problem specimens, and 8 normal skin specimens were used for KGFR staining. Paraffin sections were deparaffinized with toluene and rehydrated by serially-graded ethanol solutions. For KGFR detection, the slides were pretreated with $0.2 \%$ Triton X-100 in phosphate buffered saline (PBS) for $15 \mathrm{~min}$ at RT. After inactivation of endogenous peroxidase activity with $0.3 \% \mathrm{H}_{2} \mathrm{O}_{2}$ in methanol, the sections were preincubated with $500 \mu \mathrm{g} / \mathrm{ml}$ normal goat IgG (Sigma) and 1\% bovine serum albumin (Sigma) in PBS for one hour to block nonspecific reaction with the first antibody. Then, the sections were incubated overnight with the first antibody at $0.5 \mu \mathrm{g} / \mathrm{ml}$ anti-KGF antibody or 8,000 -fold dilution of anti-KGFR antisera, washed three times with $0.075 \%$ Brij 35 in PBS, and 
reacted with horseradish peroxidase (HRP)-goat anti-rabbit IgG (Medical \& Biological Laboratories Co., Nagoya, Japan) for one hour. After the slides were washed with $0.075 \%$ Brij 35 in PBS, HRP sites were visualized with 3, 3'-diaminobenzidine- $4 \mathrm{HCl}$ (DAB), $\mathrm{Ni}, \mathrm{Co}$ and $\mathrm{H}_{2} \mathrm{O}_{2}$ according to the method of Adams [23]. For negative control, normal rabbit IgG (Sigma) or normal rabbit serum (Sigma) was used instead of the first antibodies, respectively, in every experiment. The specimen with KGF-positive cells was KGF-positive case and the specimen without KGF-positive cells was KGF-negative case. The specimen with KGFR-positive cells was KGFR-positive case and the specimen without KGFR-positive cells was KGFR-negative case.

\section{Identification of Proliferating Cells}

The nuclear antigen associated with cell proliferation was immunohistochemically detected using anti-Ki-67 antibody (Immunotech, Marseille, France). Sections of 62 cholesteatoma specimens, 9 cavity problem specimens, and 8 normal skin specimens were used for Ki-67 staining. For this purpose, sections were first deparaffinized, then the slides autoclaved in $10 \mathrm{mM}$ citrate buffer (pH 6.0) for 15 minutes at $120^{\circ} \mathrm{C}$, according to the protocol described by Ehara et al. [24] and processed in a manner similar to that described above, except for the concentration of anti-Ki-67 antibody (1:100) and the use of HRP-goat anti-mouse $\operatorname{IgG}$ (Chemicon International, Inc, Temecula, CA) (1:100) as a second antibody. As a negative control, some sections were incubated with normal mouse IgG (Sigma) instead of anti-Ki-67 antibody. 
Identification of the type of lymphocytes

To identify the type of lymphocytes, we carried out immuno-stainings for various cell surface markers in the sections of 10 specimens of cavity problem. Sections were reacted with surface markers such as anti-CD45R0 (Dako) and anti-CD20cy antibody (Dako). A mouse monoclonal antibody against CD45R0 was used to identify activated $\mathrm{T}$ lymphocytes [25]; a mouse monoclonal antibody against CD20cy was used to identify B lymphocytes [26]. After deparaffinization, the slides were immersed with PBS, while anti-CD45R0 (5.4 $\mu \mathrm{g} / \mathrm{ml})$ and anti-CD20cy antibodies(3.55 $\mu \mathrm{g} / \mathrm{ml})$ were stained without any pretreatment. Then sections were stained by the same indirect immunohistochemistry described above. HRP-goat anti-mouse IgG (1:100) was used as the second antibody. As a negative control, some sections were incubated with normal mouse $\operatorname{IgG}$ instead of cell surface markers.

\section{Quantitative Analysis}

For quantitative analysis, more than 1,000 cells were counted in random fields at $400 \times$ magnification under light microscopy and the number of Ki-67-positive cells was expressed as a percentage of positive cells per total number of counted cells [Ki-67 labeling index (LI); mean $\pm \mathrm{SD}]$. The staining intensities of KGF and KGFR were graded as $(+)$ positive or (-) negative, compared to the background staining with normal rabbit IgG. The numbers of CD20cy-positive and CD45R0-positive cells also were expressed as a percentage of positive cells per total number of counted cells, more than 1,000 cells were counted in random fields at $400 \times$ magnification under the light microscopy [LI; mean $\pm \mathrm{SD}]$. 
Statistical Analysis

The percentage of the specimens expressing KGF and KGFR was compared between the three groups for statistical significance using the Pearson's chi-square test. The LI of Ki-67, CD20cy, and CD45R0 were expressed as mean \pm SD. Differences in LIs of Ki-67, CD20cy, and CD45R0 between each of the two groups were examined for statistical significance using the unpaired Student's $t$ test. A $P$ value less than 0.01 denoted the presence of a statistically significant difference. All analyses were performed with statistical software packages (JMP, version 8.0.2; SAS Institute Inc., Cary, NC, USA). 


\section{Results}

In 4 out of 10 cavity problem specimens (40\%), positive staining for KGF was detected in some stromal cells (KGF-positive specimen, Fig. 1Aa), while KGF staining was not detected anywhere in any slice in the remaining 6 specimens $(60 \%$, KGF-negative specimen). KGFR was detected in a small number of epithelial cells in 3 of 9 specimens (33\%, KGFR-positive case, Fig. 1Ab). Whereas, in the cholesteatoma group, 61 of 69 specimens (88.4\%) (one cholesteatoma specimen without subepithelial tissue was not used for analysis) were KGF-positive, where the stromal cells, which were mostly infiltrating lymphocytes and fibroblasts, were KGF positive (Fig. 1Ba). KGFR was detected in 37 out of 62 specimens $(59.7 \%$, KGFR-positive case) ( 8 cholesteatoma specimens without epithelium were not used for analysis), and was predominantly found in the cells of the spinous layer and also in some basal and granular cells in cholesteatoma epithelium. The staining was exclusively localized to the plasma membrane (Fig. 1Bb). In 3 of the 8 normal skin specimens (37.5\%), KGF was detected in a few stromal fibroblasts (KGF-positive, Fig. 1Ca) and KGFR was scarcely found in epithelial cells (KGFR-negative, Fig. 1Cb). In addition, when the sections were reacted with preimmune normal rabbit serum instead of the first antibody, no staining was found (data not shown).

The percentage of KGF-positive specimens in the cavity problem group was the same as that of normal skin ( $40.0 \%$ vs. $37.5 \%$, table 1, Fig. 2$)$. The percentage of KGF-positive specimens was significantly higher in the cholesteatoma group than in other cases $(88.4 \%, \mathrm{p}=0.0004$, Pearson's chi-square test, table 1, Fig. 2$)$. The incidence of KGFR-positive specimens was higher in the cavity problem group than in 
normal skin ( $33.3 \%$ vs. $0 \%$, $\mathrm{p}=0.0719$, Pearson's chi-square test, table 1 , Fig. 2$)$, but was lower than that in the cholesteatoma group $(33.3 \%$ vs. $59.7 \%, \mathrm{p}=0.1365$, Pearson's chi-square test, table 1, Fig. 2).

In specimens of the cavity problem group and in normal skin, some Ki-67-positive cells were found mainly in the basal layer of the epithelium (Fig. 1Ac, 1Cc). On the other hand, in specimens from the cholesteatoma group, Ki-67-positive cells were found mainly in the suprabasal and upper layers (Fig. 1Bc). In addition, when the sections were reacted with preimmune normal mouse IgG instead of the first antibody, no staining was found (data not shown). In cholesteatoma tissues, Ki-67-positive cells were found in the suprabasal and upper layers of the epithelium, which almost corresponded to KGFR-positive layers (data not shown). The Ki-67 LI was significantly lower in the cavity problem group than in the cholesteatoma group $(3.1 \pm 5.9 \%$ vs. $41.5 \pm 21.5 \%, \mathrm{t}=2.00, \mathrm{p}<0.0001$, Student's t-test, Table 1, Fig. 3), indicating that the proliferative activity is lower in the cavity problem group than in the cholesteatoma group.

In the specimens of the cavity problem group, CD20cy-positive cells were found in the stroma (Fig. 4A), and the CD20cy LI was significantly higher in the cavity problem group than in the cholesteatoma group $(9.5 \pm 10.8 \%$ vs. $46.9 \pm 20.1 \%$, $\mathrm{p}<0.0001$, Student's t-test, Fig. 5). On the other hand, CD45R0-positive cells were detected in the stroma of cavity problem specimens (Fig.4B) and the LI of CD45R0-positive cells was significantly lower in cavity problem group than in cholesteatoma group $(21.0 \pm 10.6 \%$ vs. $43.5 \pm 25.1 \%$, p=0.0066 Student's t-test, Fig. 5). 


\section{Discussion}

In the present study, we found that both expression and population of KGF were less in the cavity problem group than in the cholesteatoma group. The percentage of KGF-positive cases was almost the same as that of normal skin. On the other hand, the percentage of KGFR expression in the cavity problem group took a middle position between those in the cholesteatoma group and normal skin. These results suggest that the discordance of a paracrine action between stromal KGF and epithelial KGFR may be involved in the pathogenesis of a cavity problem, leading to blockage of the migration particularly in its active proliferative stage. In fact, the value of Ki-67 LI in epithelial cells in the cavity problem group was noted to be significantly lower than that in the cholesteatoma group and similar to that of normal skin.

As seen in the present results, co-expression of stromal KGF and epithelial KGFR was observed in many cholesteatoma cases, and the Ki-67 LI was higher in KGF and KGFR co-expression cases than in those without co-expression cases (12.7 $\pm 7.7 \%$ vs. $4.0 \pm 4.9 \%$; $<<0.01)[13] . \quad$ These results indicated that the paracrine action between KGF and KGFR may play an important role in the pathogenesis of cholesteatoma. On the other hand, co-expression of stromal KGF and epithelial KGFR was observed only in a few cavity problem cases (20\%: $2 / 10)$ (data not shown). And also, among the cavity problem group, the Ki-67 LI was almost the same between in those with KGF and KGFR co-expression cases and in those without co-expression cases $(5.2 \pm 5.4$ vs. $2.6 \pm 8.5)$. These results also suggested a possibility that the discordance of a paracrine action between stromal KGF and epithelial KGFR may be involved in the pathogenesis of cavity problem. 
A predominant population of chronic inflammatory cells infiltration, including lymphocytes, monocytes, and plasma cells, has been observed both in cholesteatoma and non-cholesteatomatous COM specimens in the previous study $[16,17,18]$. Among these inflammatory cells, $\mathrm{T}$ lymphocyte infiltration in the stroma and epithelium was corresponded well with KGF expression both in cholesteatoma and non-cholesteatomatous COM in our previous study [15]. In this study, we detected that CD45R0 LI was significantly lower in cavity problem cases than in cholesteatoma cases [15]. On the other hand, we also found that CD20cy-positive cells were rare both in the specimens of the cholesteatoma and non-cholesteatomatous COM group [15]. In the present study, we found that CD20cy LI was significantly higher in cavity problem cases than in cholesteatoma cases [15]. These results may point to the following two possibilities. First, the small number of infiltrating $\mathrm{T}$ cells might be correlated with lower expression of KGF, leading to impairment of epithelization and the development of troublesome granulation as seen in the cavity problem specimens. In fact, we were able to detect a highly positive correlation between the degree of inflammation expressed as T cell LI, and the levels of KGF expression both in non-cholesteatomatous and cholestetomatous COM specimens, and concluded that infiltrating T lymphocytes producing KGF may enhance the proliferative activity of epithelial cells in the COM and may facilitate cholesteatoma formation [15]. Second, a large number of infiltrating B cells might be related with the pathogenesis of the cavity problem. B cells produce antibodies to damaged tissues, and are engaged in the process of wound healing [19]. As in recent observations, chronic inflammatory diseases such as the inflammatory bowel diseases and 
rheumatoid arthritis are characterized by a local accumulation of activated B cells [20, 21,22]. Various mechanisms by which B cells are involved in the induction and maintenances of chronic inflammatory disease have been intensively investigated. As a result, it was shown that the $\mathrm{CD} 20$-positive $\mathrm{B}$ cells play roles in $\mathrm{T}$-cell activation via antigen presentation, secretion of proinflammatory cytokines, and production of self-reactive antibodies $[27,28,29,30]$. Anti-CD20 mAb rituximab is used to appreciate the various functions of B cells in the treatment of chronic inflammatory diseases [31]. So, in the cavity problem cases infiltrating B cells may play a role in granulation tissue formation.

\section{Conclusion}

From the present study, it was suggested that, in terms of the activities of cytokines such as KGF and KGFR, the pathogenesis of the cavity problem may be at a different position to that of cholesteatoma, and related in discordance rather than coordination of KGF and KGFR. It also might be caused by local imbalance of the proportion of $\mathrm{T}$ and $\mathrm{B}$ lymphocytes. The present findings are expected to be of some help in obtaining a clue to solve this intractable pathological condition. 


\section{Acknowledgement}

This study was supported in part by grants from the Naito foundation (Tokyo, Japan)

(to T. Yamamoto-fukuda). 


\section{References}

1. Portmann M. The problem of the cavity in tympanoplastic surgery. Laryngoscope $1971 ; 81: 1233-1241$.

2. Brandow EC. Surgical procedure for the mastoid cavity problem. Otolaryngol head and neck surg 1980; 88:622-624.

3. Jackson CG, Glasscock ME 3rd, Nissen AJ, Schwaber MK, Bojrab DI. Open mastoid procedures: contemporary indications and surgical technique.

Laryngoscope. 1985; 95(9 Pt 1):1037-1043.

4. Rambo JHT. Primary closure of the radical mastoidectomy wound: a technique to eliminate postoperative care. Laryngoscope 1958; 68:1216-1227.

5. Palva T. Surgery of chronic ear with cavity. Arch Otolaryngol 1963; 77:570-579.

6. Sade J. Reconstruction of the posterior canal wall in obliteration of radical mastoidectomies. Arch Otolaryngol 1963; 78:127-129.

7. Turner JL. Obliteration of mastoid cavities by musculoplasty. Laryngoscope 1966; $76: 465-471$.

8. Birzgalis AR, Farrington WT, O'Keefe L. Reconstruction of discharging mastoid cavities using the temporalis myofascial flap. Clin Otolaryngol 1994; 19:70-72.

9. Finch PW, Rubin JS, Miki T, Ron D, Aaronson SA. Human KGF is FGF-related with properties of a paracrine effector of epithelial cell growth. Science 1989; 245:752-755.

10. Koji T, Chedid M, Rubin JS, Slayden OD, Csaky KG et al. Progesterone-dependent expression of keratinocyte growth factor mRNA in 
stromal cells of the primate endometrium: keratinocyte growth factor as a progestomedin. J Cell Biol 1994; 125:393-401.

11. Werner S, Peters KG, Longaker MT, Fuller-Pace F, Banda MJ, Williams LT. Large induction of keratinocyte growth factor expression in the dermis during wound healing. Proc Natl Acad Sci 1992; 89:6896-6900.

12. Staiano-Coico BL, Kruegter JG, Rubin JS, D’limi S, Vallat VP, Valentino L, et al. Human keratinocyte growth factor effects in a porcine model of epidermal wound hearing. J Exp Med 1993; 178:865-878.

13. Yamamoto-Fukuda T, Aoki D, Hishikawa Y, Kobayashi T, Takahashi H, Koji T. Possible involvement of keratinocyte growth factor and its receptor in enhanced epithelial-cell proliferation and acquired recurrence of middle-ear cholesteatoma. Lab Invest 2003; 83:123-136.

14. Yamamoto-Fukuda T, Terakado M, Hishikawa Y, Koji T, Takahashi H. Topical application of 5-fluorouracil on attic cholesteatoma results in downregulation of keratinocyte growth factor and reduction of proliferative activity. Eur Arch Otorhinolaryngol 2008; 265:1173-1178.

15. Yamamoto-Fukuda T, Takahashi H, Terakado M, Hishikawa Y, Koji T. Expression of keratinocyte growth factor and its receptor in noncholesteatomatous and cholesteatomatous chronic otitis media. Otol Neurotol 2010; 31:745-751.

16. Sculerati N, Bluestone CD. Pathogenesis of cholesteatoma. Otolaryngol Clin North Am 1989; 22:859-868.

17. Sudhoff H, Bujia J, Fisseler-Eckhoff A, Holly A, Schulz-Flanke C, Hildmann H. Expression of a cell-cycle-associated nuclear antigen (MIB 1) in cholesteatoma 
and auditory meatal skin. Laryngoscope 1995; 105:1227-1231.

18. Wright CW, Meyerhoff WM. Pathology of otitis media. Ann Otol Rhinol Laryngol 1994; 103:24-26.

19. Nishino N, Ito S, Suzuki H, Isobe K. Antibodies to wound tissue enhance cutaneous wound hearing. Immunology 2009; 128:369-380.

20. Brandtzaeg P. Role of immune system-dangers of a nonholistic approach in explaining health and disease. In: Gugpenheim VB, eds. Periodontology Today. Switzerland: Karger Basel; 1988:196.

21. Ziff M. Emigration of lymphocytes in rheumatoid synovitis. Aduan Inflam Res $1988 ; 12: 1$

22. Elson CO. The immunology of inflammatory bowel disease. In: Kirsner JB, Shorter RG, eds. Inflammatory Bowel Disease. Philadelphia, PA: Lea and Febiger; 1988:97.

23. Adams JC. Heavy metal intensification of DAB-based HRP reaction product. J Histochem Cytochem 1981; 29:775.

24. Ehara H, Koji T, Deguchi T, Yoshii A, Nakano M, Nakane PK, et al. Expression of estrogen receptor in diseased human prostate assessed by non-radioactive in situ hybridization and immunohistochemistry. Prostate 1995; 27:304-313.

25. Norton AJ, Ramsay AD, Smith SH, Beverley PC, Isaacson PG. Monoclonal antibody (UCHL1) that recognises normal and neoplastic T cells in routinely fixed tissues. J Clin Pathol 1986; 39:399-405.

26. Cartun RW, Coles FB, Pastuszak WT. Utilization of monoclonal antibody L26 in the identification and confirmation of B-cell lymphomas. A sensitive and specific 
marker applicable to formalin-and B5-fixed, paraffin-embedded tissues. Am J Pathol 1987; 129:415-421.

27. Mason D. The role of B cells in the programming of T cells for IL-4 synthesis. J Exp Med 1996; 183:717-719.

28. Martin F, Chan AC. Pathogenic roles of B cells in human autoimmunity; insights from the clinic. Immunity $2004 ; 20: 517-527$.

29. Bombardieri M, Barone F, Humby F, Kelly S, McGurk M, Morgan P, et al. Activation-induced cytidine deaminase expression in follicular dendritic cell networks and interfollicular large B cells supports functionality of ectopic lymphoid neogenesis in autoimmune sialoadenitis and MALT lymphoma in Sjogren's syndrome. J Immunol 2007; 179:4929-4938.

30. Timmer TC, Baltus B, Vondenhoff M, Huizinga TW, Tak PP, Verweij Cl, et al. Inflammation and ectopic lymphoid structures in rheumatoid arthritis synovial tissues dissected by genomics technology: identification of the interleukin-7 signaling pathway in tissues with lymphoid neogenesis. Arthritis Rheum 2007; $56: 2492-2502$.

31. Nagel A, Hertl M, Eming R. B-cell-directed therapy for inflammatory skin diseases. J Invest Dermatol 2009; 129:289-301. 
Table 1. Positive rate of KGF, KGFR and mean of Ki-67 LI, CD20cy, CD45R0 in cavity problem, cholesteatoma and normal skin specimens

\begin{tabular}{|c|c|c|c|c|c|c|c|}
\hline & $\begin{array}{c}\text { Number } \\
\text { (Male/Female) }\end{array}$ & $\begin{array}{l}\text { Age } \\
\text { (Yr) }\end{array}$ & Ki-67 LI & $\begin{array}{c}\text { KGF-Positive } \\
\text { Cases (\%) }\end{array}$ & $\begin{array}{l}\text { KGFR-Positive } \\
\text { Cases (\%) }\end{array}$ & CD20cy LI & CD45R0 LI \\
\hline Cavity Problem & $10(6 / 4)$ & 52 & $3.1 \pm 5.9$ & $4 / 10(40.0)$ & $3 / 9(33.3)$ & $46.9 \pm 20.1$ & $21.0 \pm 10.6$ \\
\hline Cholesteatoma & $70(40 / 30)$ & 42 & $41.5 \pm 21.5$ & $61 / 69(88.4)$ & $37 / 62(59.7)$ & $9.5 \pm 10.8$ & $43.5 \pm 25.1$ \\
\hline Normal Skin & $8(4 / 4)$ & 49 & $0.7 \pm 0.4$ & $5 / 8(37.5)$ & $0 / 7(0)$ & & \\
\hline
\end{tabular}




\section{Figure Legend}

Figure 1.

Aa-Ac: Immunohistochemical detections in paraffin sections of a cavity problem specimen. Aa, Immunohistochemistry using anti-KGF antibody. Staining for KGF was detected in some stromal cells. Ab, Immunohistochemistry using anti-KGFR serum. KGFR was weakly detected in the epithelium. Ac, A small number of Ki-67-positive cells in basal and spinous layers of the epithelium. Arrows: positive cells.

Ba-Bc: Immunohistochemical detections in paraffin sections of a cholesteatoma specimen. Ba, Immunohistochemistry using anti-KGF antibody. Intense staining for KGF was detected in many stromal cells. $\quad \mathrm{Bb}$, Immunohistochemistry using anti-KGFR serum. KGFR-positive cells were predominantly detected in the basal, spinous, and granular layers of the epithelium. KGFR-positive cells were almost correspondent to Ki-67-positive cells. Bc, Ki-67-positive cells in the suprabasal and upper layers of the epithelial lesion. Arrows: positive cells, Asterisks: positive layers. (reproduced with modification with permission from T. Yamamoto-Fukuda et al. [15])

Ca-Cc: Immunohistochemical detections in paraffin sections of normal skin. Ca, Immunohistochemistry using anti-KGF antibody. KGF-positive cells are detected in few stromal cells. Cb, KGFR-positive cells are scarcely found except for weak staining within hair follicles (arrows). Cc, Ki-67-positive cells are detected in the basal layer. Arrows: positive cells, magnification 350x. (reproduced with modification, with permission from T. Yamamoto-Fukuda et al. [13]) 
Figure 2.

Positive rates of KGF and KGFR expression in cavity problem, cholesteatoma and normal skin specimens. Black bars indicate KGF-positive cases and white bars indicate KGFR-positive cases. ${ }^{*}: p=0.0004,{ }^{* *}: p=0.0719,{ }^{* * *}: p=0.1365$.

Figure 3.

Ki-67 labeling index (LI) in cavity problem, cholesteatoma and normal skin specimens. Data are mean $\pm \mathrm{SD} . \quad *: P<0.0001$.

Figure 4.

Immunohistochemical detections of CD20cy and CD45R0 in serial sections of a cavity problem specimen. A, Immunohistochemistry using anti-CD20cy antibody. $\mathrm{B}$, Immunohistochemistry using anti-CD45R0 antibody. C, The section was reacted with normal mouse $\operatorname{IgG}$ instead of the first antibody as a negative control and no staining was found.

Arrows: positive cells.

Figure 5.

LI of CD20cy and CD45R0 in cavity problem and cholesteatoma specimens. The cholesteatoma data were reproduced with modification, with permission from $\mathrm{T}$. Yamamoto-Fukuda et al. [15]. Data are mean \pm SD. $\quad *: P<0.0001, * *: P=0.0066$. 
Figure 1

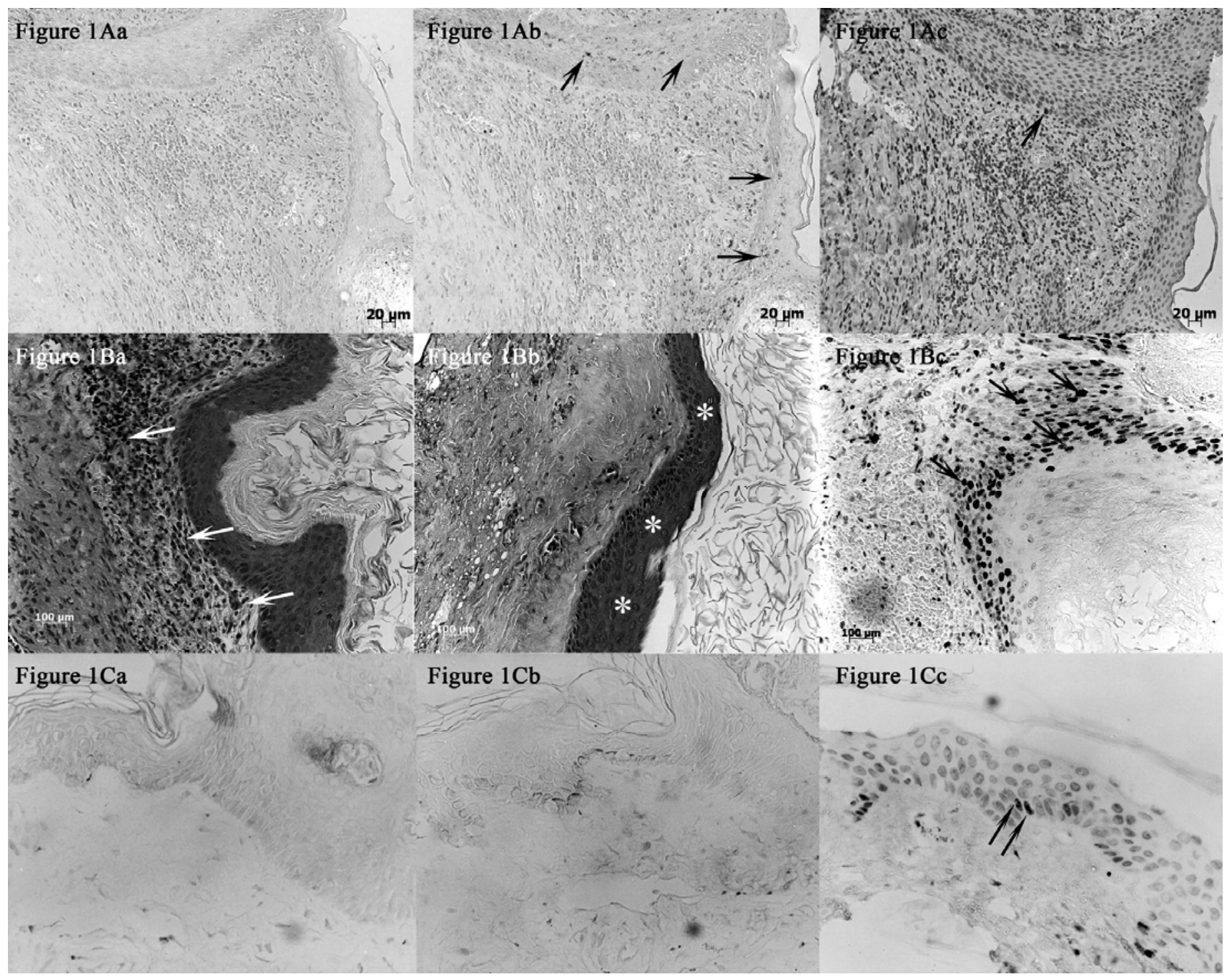


Figure 2

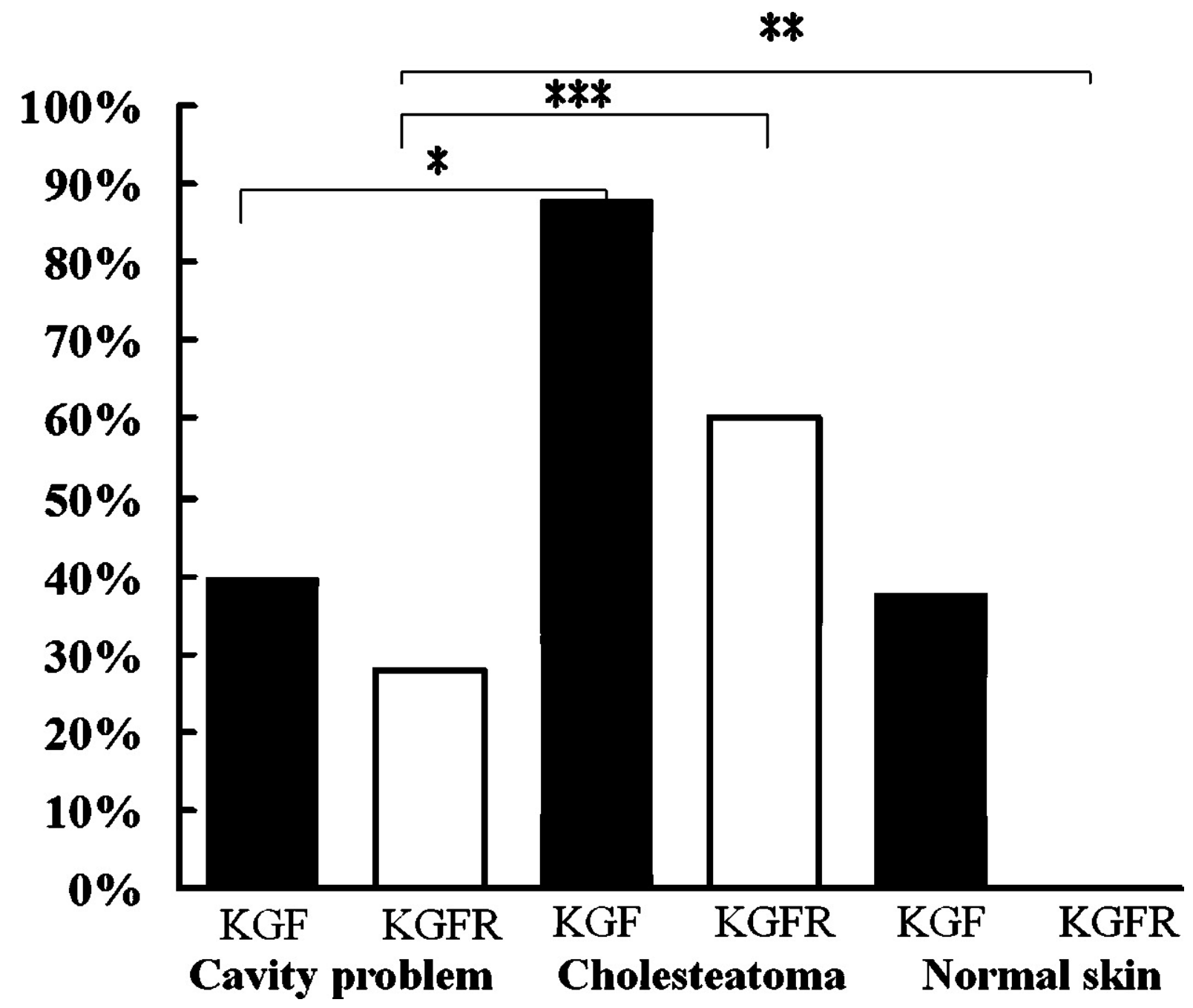


Figure 3

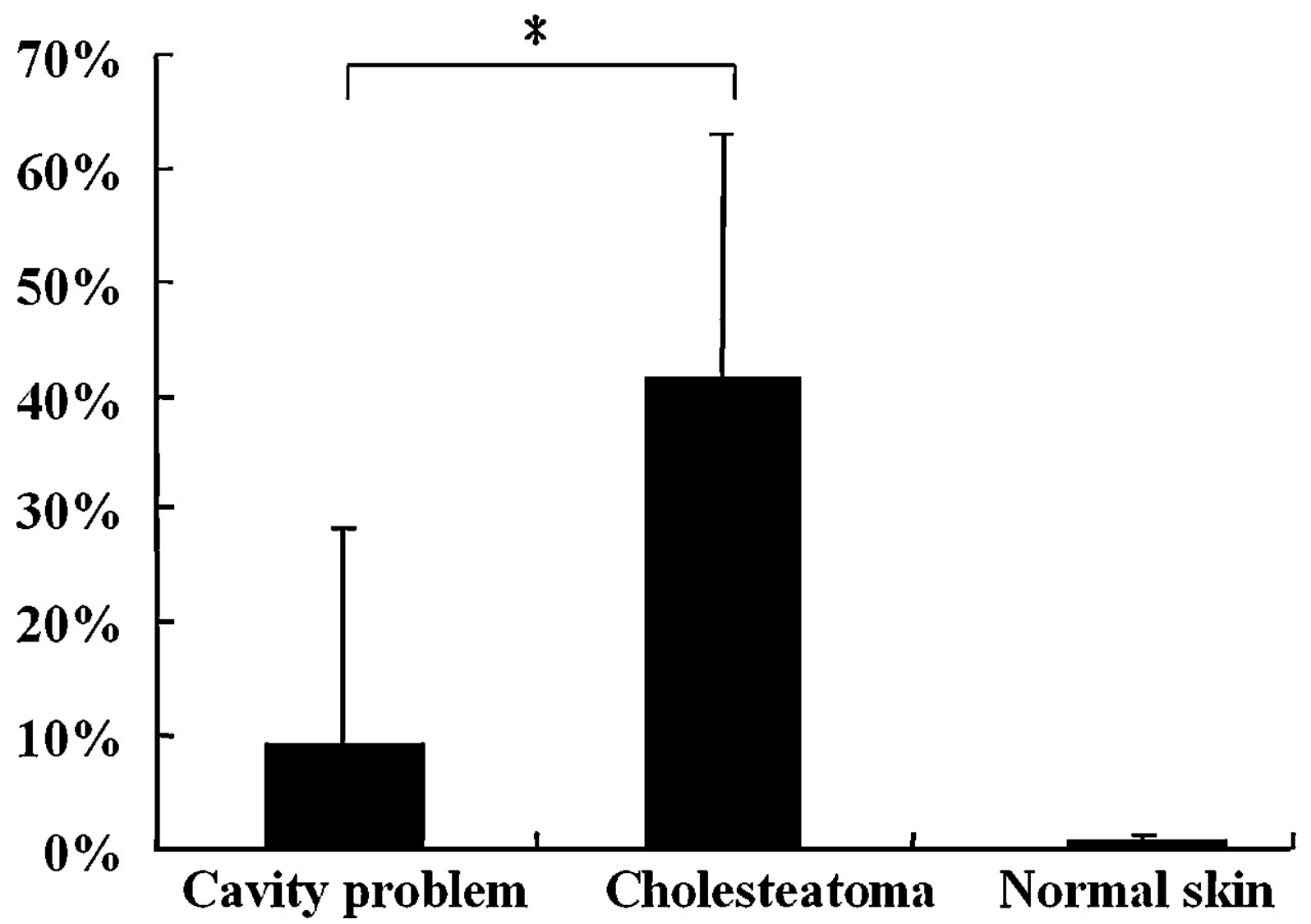


Figure 4
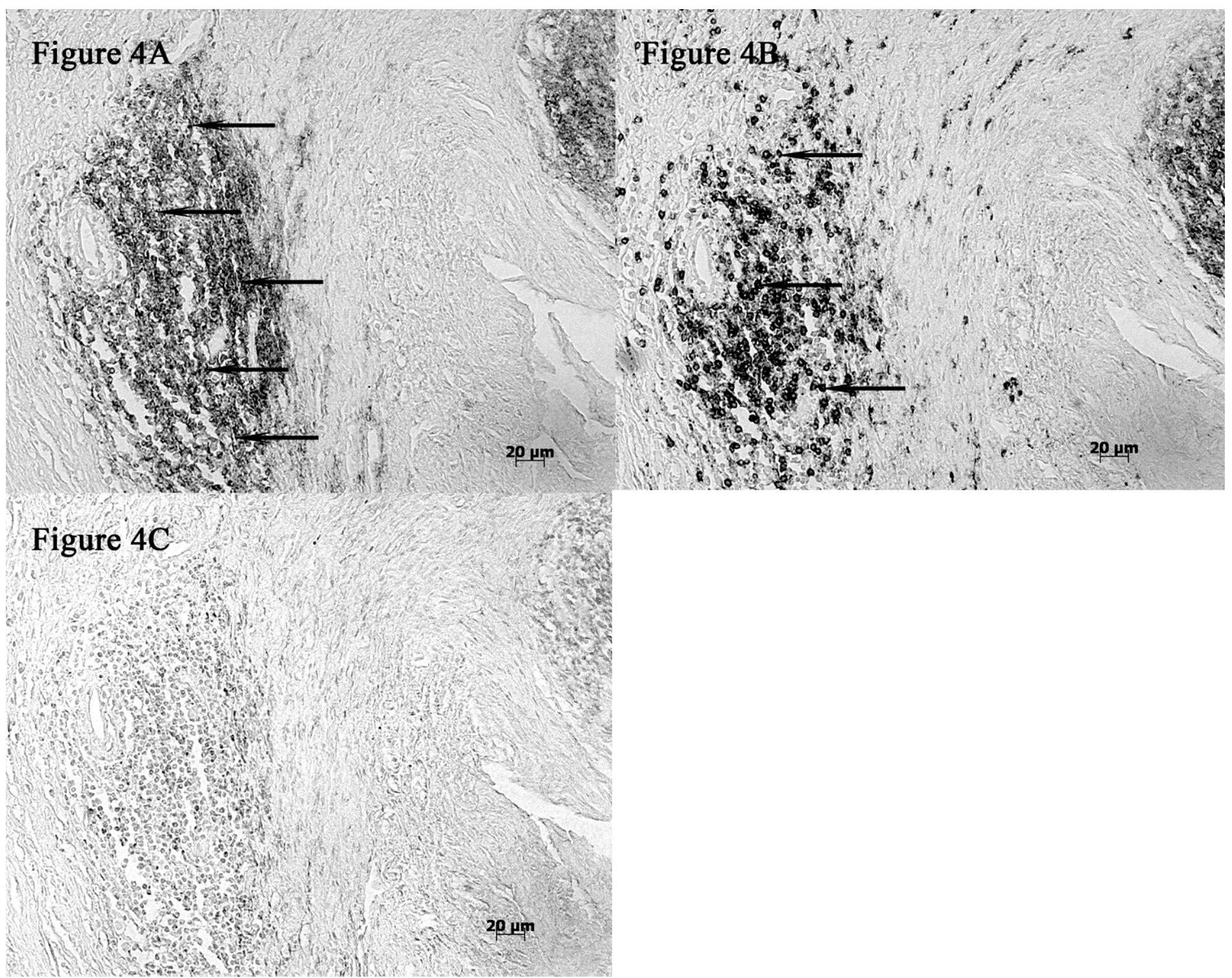